\title{
Influential Node Control Strategy for Opinion Evolution on Social Networks
}

\author{
Cheng Ju, ${ }^{1}$ Jinde Cao, ${ }^{1,2}$ Weiqi Zhang, ${ }^{3}$ and Mengxin Ji ${ }^{4}$ \\ ${ }^{1}$ Department of Mathematics, Southeast University, Nanjing 210096, China \\ ${ }^{2}$ Department of Mathematics, Faculty of Science, King Abdulaziz University, Jeddah 21589, Saudi Arabia \\ ${ }^{3}$ School of Computer and Science and Engineering, Southeast University, Nanjing 210096, China \\ ${ }^{4}$ College of Economics, Zhejiang University, Hangzhou 310058, China
}

Correspondence should be addressed to Jinde Cao; jdcao@seu.edu.cn

Received 16 October 2013; Accepted 17 November 2013

Academic Editor: Qiankun Song

Copyright (c) 2013 Cheng Ju et al. This is an open access article distributed under the Creative Commons Attribution License, which permits unrestricted use, distribution, and reproduction in any medium, provided the original work is properly cited.

\begin{abstract}
We study opinion dynamics in social networks and present a new strategy to control the invasive opinion. A developed continuousopinion evolution model is proposed to describe the mechanism of making decision in closed community. Two basic strategies of evolution are determined, and some basic features of our new model are analyzed. We study the different invasive strategies. It is shown via using Monte Carlo simulations that our new model shows different invulnerability with traditional model. Node degree and cohesion in invasive small-world community plays less significant role when the evolution of opinion is continuous rather than dichotomous. Using simulation, we find one kind of Influential Nodes that can affect the outcome dramatically, while these Influential Nodes are sensitive to their node degree and the evolution weight. Thus, we develop invasive control strategy based on these features.
\end{abstract}

\section{Introduction}

There is long history in social science to learn social phenomenon such as opinion cluster and invasion. Some simple mathematic models are helpful tools to simulate such phenomenon. Recently the mathematic models learning the invasion and spread of new opinion have received a lot of attention [1-3]. At present, the spread rumors in real world become easier and can make huge influence on our daily life $[4,5]$. Thus, the study of the dynamics of different opinions and rumors has gradually become one of the most popular fields in the study of complex networks $[6,7]$.

Some other questions come out with gradually deepening the study of rumors spread. It is known that some rumors and ideas seem harmless at first while they can do great harm to the health of the network if they are not controlled. To deal with such problem, many researches developed lots of strategies [8-12]. Community structure appears to be essential for the evolution of invasive opinion [13, 14]. Some researches focus on a few nodes which have large connective like Targeted immunization [11]. Furthermore, some researches also use real world data and cases to develop and evaluate their control strategy [15-17].

In order to understand the role of the Influential Node and the different strategies of opinion evolutions in the opinions dynamics, for simplicity, a continuous-opinion model is proposed in this paper, including two strategies of opinion evolution. It does not aim at an exact description of reality. However, it focuses on discovering some essential and fundamental features of an otherwise very complex and multiple phenomena by doing some crude approximations. Therefore, we use Monte Carlo method to simulate the reality and determine the sensitivity of the model and our new strategy in the different situation.

In this paper, to understand the difference between traditional dichotomous model and our new continues model and the mechanism of the Influential Node in the opinions dynamics, we focus on the following four questions. First of all, how can the different evolution strategies affect the dynamic of the model? Are there any transition points where the model is very sensitive? The answer can help us better understanding the dynamic of this continues-opinion 
evolution model. Then we would like to know how the size of the invasive community and its node degree can contribute to the outcome of the model when the proportion of the strategies is fixed. Some feature of network in former study [18] may not be suitable for continues model. It is also known that the community structure can affect the evolution. So could the community structure affect the evolution of the network? If so, is the effect the same as that in the traditional dichotomous model? Last, in order to know the role it plays in the evolution, it is critical to know how Influential Node can contribute to the evolution of the network and are such nodes sensitive to some factors such as node degree. If we have better understanding of this kind of nodes, we could develop new strategy to control the spread of invasive rumor.

In the first part, we investigate the outcome of the model with different proportion of the opinion evolution strategy. The first strategy of evolution is the change of the topology structure of the network, and the second strategy is the intersecting opinion of the different points in the network. The different proportion of two basic strategies will lead to the different outcome of the dynamic model. If the model only uses the first way, the opinion of every node will not change, while the topology structure of the network will change all the time. Hence, the entire network will evolute into several small communities, and there should be no intersects between two different networks in certain time. When the model only uses the second way, the opinion of different nodes will change while the topology structure will remain the same. In this situation, the model will develop into single connected network where the structure remains the same. We define the term "consensus": if the value of every node in the network converge to a certan interval, it means this model makes "consensus". Using Monte Carlo method, we simulate the situation for different length of time. We count the probability of consensus for every $1 \%$ change of the proportion of the two strategies.

In the second part, we focus on some other questions. At first, we assign the same value of opinion to all the nodes in the network. Then we develop two major kinds of invasion for the contrary opinion. The first way is invasion with smallworld community. Some former studies show that the size of community and the node degree are two major factors for the outcome. Therefore, we simulate the outcome with different size of invasive small-world and its node degree. We define Influential Node in opinion invasion. This kind of nodes has more node degree and better stability. Then we study the different factors which can affect the power of Influential Node and develop new control strategy to impede the spread of invasive opinion.

\section{Model Description}

2.1. Symbol Description of Network. We develop our continues-opinion model based on our former discrete multiopinion model [19].

2.2. Two Strategies of the Evolution. For the first strategy, the opinion of every node will not change, while the topology structure of the network will change every step. Hence, if
TABLE 1: Symbols table.

\begin{tabular}{ll}
\hline Symbol & Description \\
\hline$N$ & The number of nodes in the entire network \\
$M$ & The number of Influential Nodes \\
$M N$ & The proportion of the invasive nodes \\
$K$ & The number of invasive nodes \\
& The average number of edges for every node (average \\
$m$ & node degree) \\
$k$ & The average number of extra edges for the Influential \\
$P$ & The average number of extra edges for the nodes in the \\
$t$ & Thvasive community \\
$T$ & Time (or the number of the steps) \\
$w$ & The length of experiment \\
$d_{i j}(t)$ & $\begin{array}{l}\text { If nodes } i \text { and } j \text { connect at time } t d_{i j}(t)=1 . \text { Otherwise } \\
d_{i j}(t)=0\end{array}$ \\
$\sigma_{i}(t)$ & The opinion value of node $i$ at time $t$
\end{tabular}

the node $i$ connects with the node $j$ and their opinions are different, the node $i$ will remove the edge to the $j$ and randomly choose. And the node $i$ will then find a note which has the same opinion to develop a new edge.

For the second strategy, the opinions are intersected with the different connected nodes in the network. In some former work, the network is dichotomous. Therefore, the process of this strategy can be expressed as a differential equation as follows (see Table 1 for the description of the symbols):

$$
\sigma_{i}(t+1)=\operatorname{sign}\left[\sum_{j=1}^{N} d_{i j} \sigma_{i}(t) \xi_{i j}(t)+h_{i}(t)\right] .
$$

However, in real world, new kind of invasive opinion may intersect with traditional opinion and therefore develop new kind of opinion. For example, some people would have neutralized opinion when facing two extreme opinions. Therefore, we propose our continues-opinion model as follows:

$$
\sigma_{i}(t+1)=(1-w)\left[\sum_{j=1}^{N} d_{i j} \sigma_{j}(t)\right]+w\left[\sigma_{i}(t)\right] .
$$

It reflects the fact that for every node, its own opinion and the opinion of its connected nodes can contribute to its opinion in the future. The difference is that, in this paper, extreme opinions can be neutralized and develop to new opinion.

\section{The Sensitivity of the Model to the Proportion of Two Strategies}

In order to determine how the proportion of two strategies can affect the evolution, we stimulate by using Monte Carlo method. 


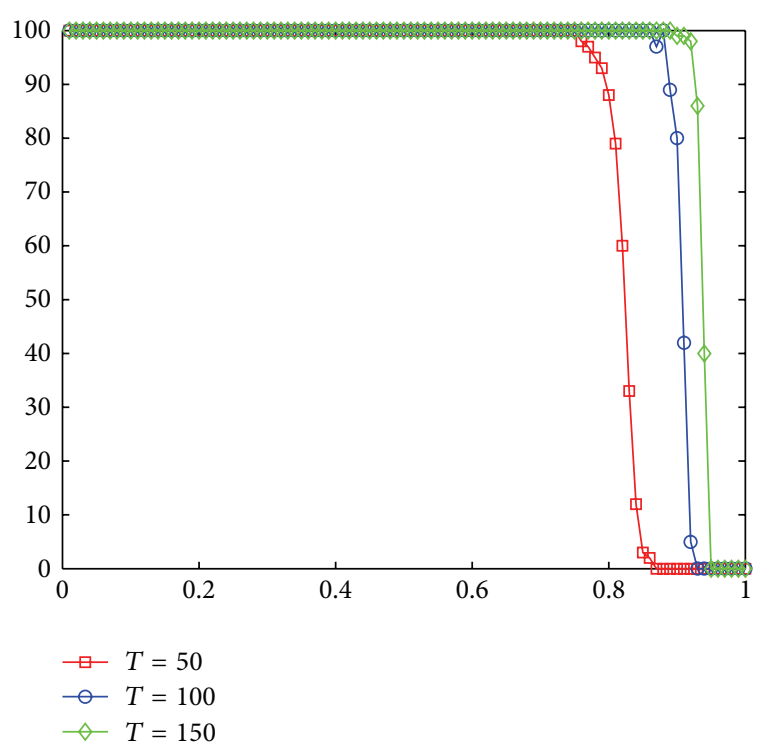

FIgURE 1: The $y$-axis represents the number of win in 100 experiments. The $x$-axis represents the proportion of two strategies.

First of all, we define "consensus" that if at the end of experiment, every in the network converges to an interval and the range of this interval is less than 0.2 . We consider the situation that $N=1000$ and the initial value of every node is obeying uniform distribution on $[-1,1]$. We simulated the probability of consensus with different proportion of two strategies.

Figure 1 shows that, compared with traditional model, nodes in continues-model are more likely to make consensus. We can see that the probability for consensus is high when the proportion of first strategy is little. The probability for consensus decreased sharply by the increase of the proportion after $P>0.8$. Hence, in conclusion, we determine that the phase transition point is around 0.85 when $T=50$ and will increase a little with the increase of $T$.

\section{Evolution under Small-World Invasive Strategy}

Some researches study some features of small-world community. Connection of the community can make the node in it prefer grouping together and therefore make critical impact on opinion evolution in the entire network [18].

First, we construct a scale-free network and the value of every node is 1 . Then the invasive nodes become -1 . In order to define the outcome of the invasion, we define the term "strong win" if all values of the nodes in the network converge to $[-1,-0.75]$ and "weak win" if all the values of the nodes are smaller than 0 .

We consider the situation that $N=1000$ and $M=0.4$, 0.42 , and 0.45 . Then we randomly add some extra nodes into invasive community. Therefore, we can have a small-world invasive community.

Figure 2 shows the probability of weak win for different node degree in invasive community. When $M=0.45$ in

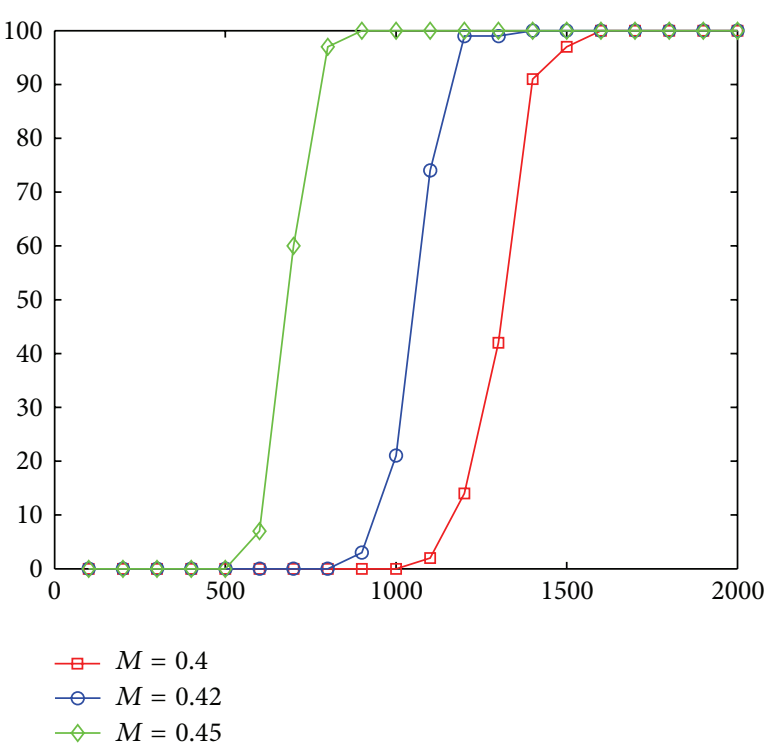

FIGURE 2: The $y$-axis represents the number of weak win in 100 experiments. The $x$-axis represents $k$.

new model, if invasive community wants to have "weak win," it should have greater average node degree compared with traditional model. Although node degree still can help minority succeed, the effect is much smaller.

Remark 1. In continues-opinion evolution model, smallworld communities are less likely to success. They need much dense of connection to maintain their impact on the network. Moreover, size of community plays more significant role in evolution. In other word, the communities with large size are more likely to success.

\section{Evolution under Influential Node Invasive Strategy}

It is known that in the real network, some celebrities can make far more impact to the opinion evolution compared with normal people. Therefore, we present a kind of node to simulate such people. This kind of node has two main characteristics.

(1) They have much more node degrees compared with other nodes.

(2) They are less likely to change their own opinion value compared with ordinary nodes.

In order to have better understanding of this kind of nodes, we investigate three factors of such nodes:

(1) quantity: $n$

(2) Their influence (their node degree): $m$

(3) how can they be influenced by other nodes: $w$.

Therefore, we simulate with different parameters to find out how these three factors can affect the success rate for the invasive community. 


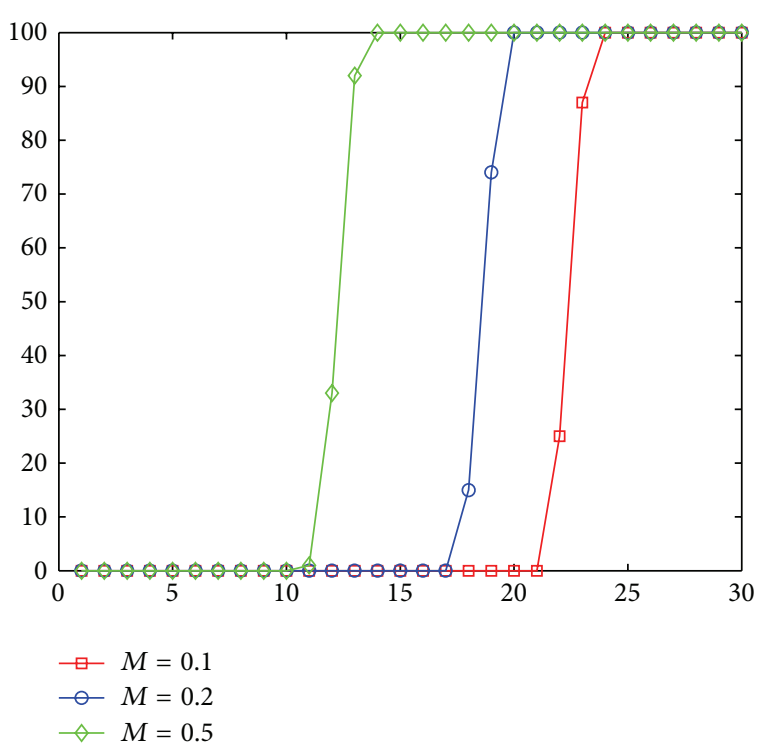

FIgURE 3: The $y$-axis represents the number of strong win in 100 experiments. The $x$-axis represents $n$, when $m=50$.

5.1. The Effect of the Number of Influential Nodes. We consider the situation that $N=1000, w=1, m=50$, and $M=0.1$, 0.2 , and 0.5 . Then we randomly choose $N M$ nodes in invasive nodes and $n$ invasive nodes as Influential Nodes. We do 100 experiments at different $n$ and find the probability for invasive community to win.

Figure 3 shows the probability for invasive community to win with different number of Influential Nodes. It shows that the outcome is sensitive to the number of Influential Nodes. When $M=0.1, n<10$, there seems to be no chance for invasive community to win. However, the probability increases abruptly around $n=12$. The community with smaller size may need more Influential Nodes to keep success. Hence, near the transition point, controlling the number of the Influential Nodes is an economic and efficient way to control the invasive opinion.

Remark 2. The spread power of invasive opinion is sensitive to the number of Influential Nodes. To control the spread of invasive opinion, controlling the number of Influential Nodes is an economic and efficient strategy when $n$ is not large.

5.2. The Effect of the Degree of Influential Nodes. We consider the situation that $N=1000, w=1, n=10$, and $M=$ $0.3,0.4$, and 0.5 . Then we randomly choose $N M$ nodes in invasive nodes and $n$ invasive nodes as Influential Nodes. We do 100 experiments with different node degree and find the probability for invasive community to win.

Figure 4 shows the probability for invasive community to win with different node degree. It is known that the celebrities with more influence and more followers can better influence the opinion evolution. In our model, we define the influence as node degree. In this simulation we find that the model is also sensitive to the node degree of Influential Nodes. With low degree, invasive community still has very small chance to

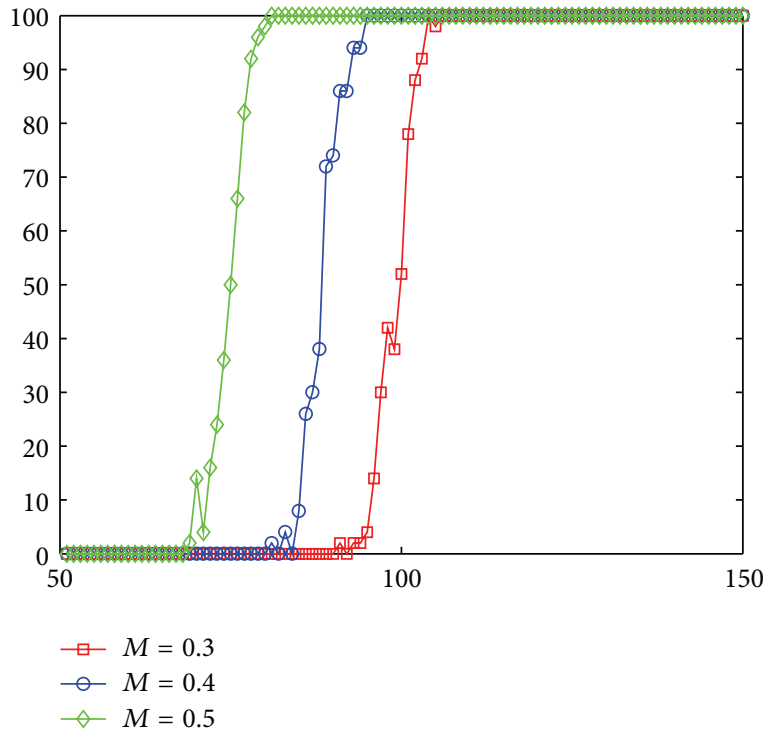

FIGURE 4: The $y$-axis represents the number of strong win in 100 experiments. The $x$-axis represents $m$, when $n=10$.

win. However, with the increase of degree, Influential Nodes can play much greater impact on evolution.

Remark 3. The spread power of invasive opinion is sensitive to the degree of Influential Nodes. To control the spread of invasive opinion, controlling the degree of Influential Nodes is an economic and efficient strategy when $n$ cannot be controlled.

5.3. The Effect of the Evolution Weight of Influential Nodes. In real world social network, evolution weight for every single point can be considered as the influence of this individual. The nodes with great weight can make more impact on the opinion evolution.

We consider the situation that $N=1000, w=1, n=$ 15 , and $M=0.45$. Then we randomly choose $N M$ nodes in invasive nodes and $n$ invasive nodes as Influential Nodes. We do 100 experiments with different evolution weight $w$.

In our pretreatment, we find that if $w<1$, the nodes in entire network would never converge to the interval $[-1,-0.75]$. Therefore, we try to use Convergence Interval Midpoint to evaluate the impact of Influential Nodes on the evolution.

Figure 5 shows the Convergence Interval Midpoint for every experiment. It shows that with the decrease of $w$, the Convergence Interval Midpoint approaches to 0 abruptly. Though it still can make negative influence on the entire network, the loss would be reduced a lot. Even a little reduction of $w$ can contribute a lot to control the harm of the invasion.

Remark 4. The opinion evolution is sensitive to the evolution weight of Influential Nodes. Although reducing $w$ cannot control the spread of invasive opinion, it can reduce the negative impact on the entire network. Therefore it can help the entire network recover much easier. 


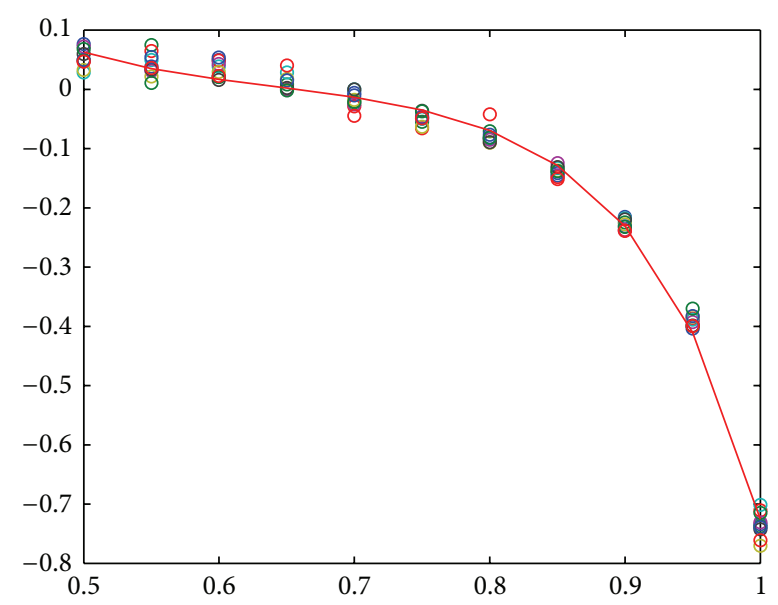

Figure 5: The $y$-axis represents the Convergence Interval Midpoint for every experiment. The $x$-axis represents $w$ when $n=15, m=$ 50 , and $M=0.45$.

\section{Conclusions}

In conclusion, we have investigated a developed continuesopinion evolution model. Two basic strategies of evolution are determined, and some basic features of our new model are analyzed. Then we analyze Influential Node and its impact on entire network. Different invasive opinion control strategies are also considered. By analyzing the phase transition point, we find that our new model is more likely to make consensus compared with the discrete traditional model. Our model can better characterize the process of the opinion neutralization. In continues model, invasive small-world community is less likely to achieve success. In other words, community size is much more significant in this new model. Further analysis shows that one kind of nodes can play critical role in invasion. With a little proportion of Influential Nodes, invasive community can achieve success even if the size is small. Hence we investigate three factors which may affect the outcome: quantity, node degree, and evolution weight. It has been observed that Influential Nodes are sensitive to their population and node degree. Though the evolution weight cannot contribute to controlling the spread of invasion, it can reduce the negative impact of invasion. Therefore, controlling these three parameters of Influential Nodes can be an effective method to impede the invasion and reduce the potential loss.

\section{Conflict of Interests}

The authors declare that there is no conflict of interests regarding the publication of this paper.

\section{Acknowledgments}

This work was jointly supported by the National Natural Science Foundation of China under Grants nos. 61272530 and 11072059, the Natural Science Foundation of Jiangsu Province of China under Grant no. BK2012741, and the Specialized
Research Fund for the Doctoral Program of Higher Education under Grant nos. 20110092110017 and 20130092110017.

\section{References}

[1] N. Crokidakis and F. L. Forgerini, "Consequence of reputation in the Sznajd consensus model," Physics Letters A, vol. 374, no. 34, pp. 3380-3383, 2010.

[2] P. Holme and M. E. J. Newman, "Nonequilibrium phase transition in the coevolution of networks and opinions," Physical Review E, vol. 74, no. 5, Article ID 056108, 5 pages, 2006.

[3] R. Lambiotte, M. Ausloos, and J. A. Hołyst, "Majority model on a network with communities," Physical Review E, vol. 75, no. 3, Article ID 030101, 4 pages, 2007.

[4] F. Radicchi and S. Fortunato, "Explosive percolation: a numerical analysis," Physical Review E, vol. 81, no. 3, Article ID 036110, 10 pages, 2010.

[5] M. Girvan and M. E. J. Newman, "Community structure in social and biological networks," Proceedings of the National Academy of Sciences of the United States of America, vol. 99, no. 12, pp. 7821-7826, 2002.

[6] P. L. Krapivsky and S. Redner, "Dynamics of majority rule in two-state interacting spin systems," Physical Review Letters, vol. 90, no. 23, Article ID 238701, 4 pages, 2003.

[7] M. Lewenstein, A. Nowak, and B. Latané, "Statistical mechanics of social impact," Physical Review A, vol. 45, no. 2, pp. 763-776, 1992.

[8] Y. Chen, G. Paul, S. Havlin, F. Liljeros, and H. E. Stanley, "Finding a better immunization strategy," Physical Review Letters, vol. 101, no. 5, Article ID 058701, 4 pages, 2008.

[9] R. Cohen, K. Erez, D. Ben-Avraham, and S. Havlin, "Resilience of the Internet to random breakdowns," Physical Review Letters, vol. 85, no. 21, pp. 4626-4628, 2000.

[10] R. Cohen, K. Erez, D. Ben-Avraham, and S. Havlin, "Breakdown of the internet under intentional attack," Physical Review Letters, vol. 86, no. 16, pp. 3682-3685, 2001.

[11] P. Holme, B. J. Kim, C. N. Yoon, and S. K. Han, "Attack vulnerability of complex networks," Physical Review E, vol. 65, no. 5, Article ID 056109, 14 pages, 2002.

[12] L. K. Gallos, F. Liljeros, P. Argyrakis, A. Bunde, and S. Havlin, "Improving immunization strategies," Physical Review E, vol. 75, no. 4, Article ID 045104, 4 pages, 2007.

[13] M. E. J. Newman, "Scientific collaboration networks. I. Network construction and fundamental results," Physical Review E, vol. 64, no. 1, Article ID 016131, 8 pages, 2001.

[14] M. E. J. Newman, "Scientific collaboration networks. II. Shortest paths, weighted networks, and centrality," Physical Review E, vol. 64, no. 1, Article ID 016132, 7 pages, 2001.

[15] R. M. Tripathy, A. Bagchi, and S. Mehta, "A study of rumor control strategies on social networks," in Proceedings of the ACM 19th International Conference on Information and Knowledge Management (CIKM '10), pp. 1817-1820, ACM, October 2010.

[16] A. J. Kimmel and A. Audrain-Pontevia, "Analysis of commercial rumors from the perspective of marketing managers: rumor prevalence, effects, and control tactics," Journal of Marketing Communications, vol. 16, no. 4, pp. 239-253, 2010.

[17] L. Zhao, Q. Wang, J. Cheng, Y. Chen, J. Wang, and W. Huang, "Rumor spreading model with consideration of forgetting mechanism: a case of online blogging LiveJournal," Physica A, vol. 390, no. 13, pp. 2619-2625, 2011. 
[18] G. Huang, J. Cao, and Y. Qu, “The minority's success under majority rule," Physica A, vol. 388, no. 18, pp. 3911-3916, 2009.

[19] C. Ju, Y. Cao, and P. Zhou, "The effect of different strategies and the structure on opinion formation," Discrete Dynamics in Nature and Society, vol. 2013, Article ID 705318, 6 pages, 2013. 


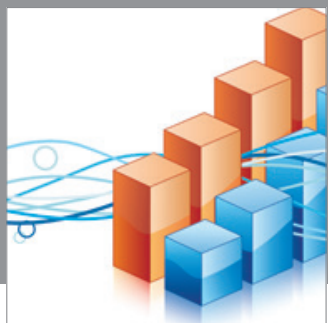

Advances in

Operations Research

mansans

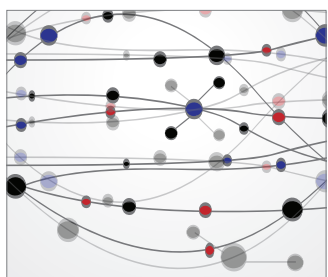

The Scientific World Journal
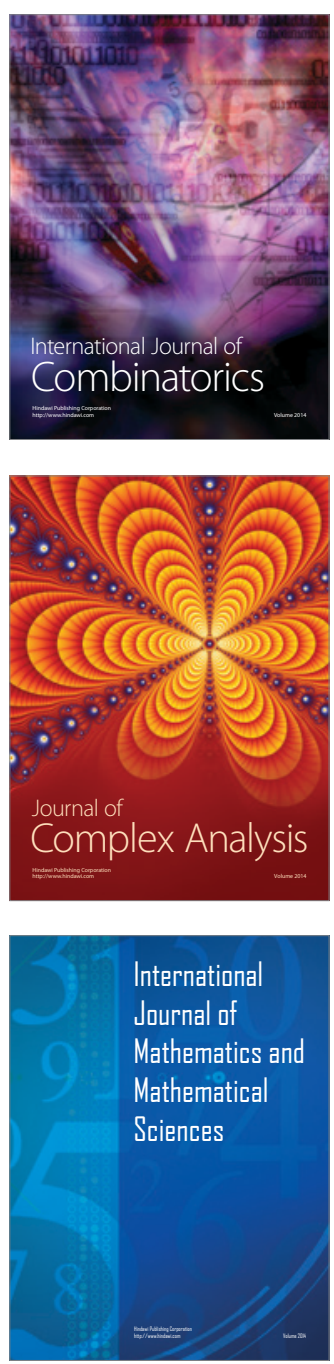
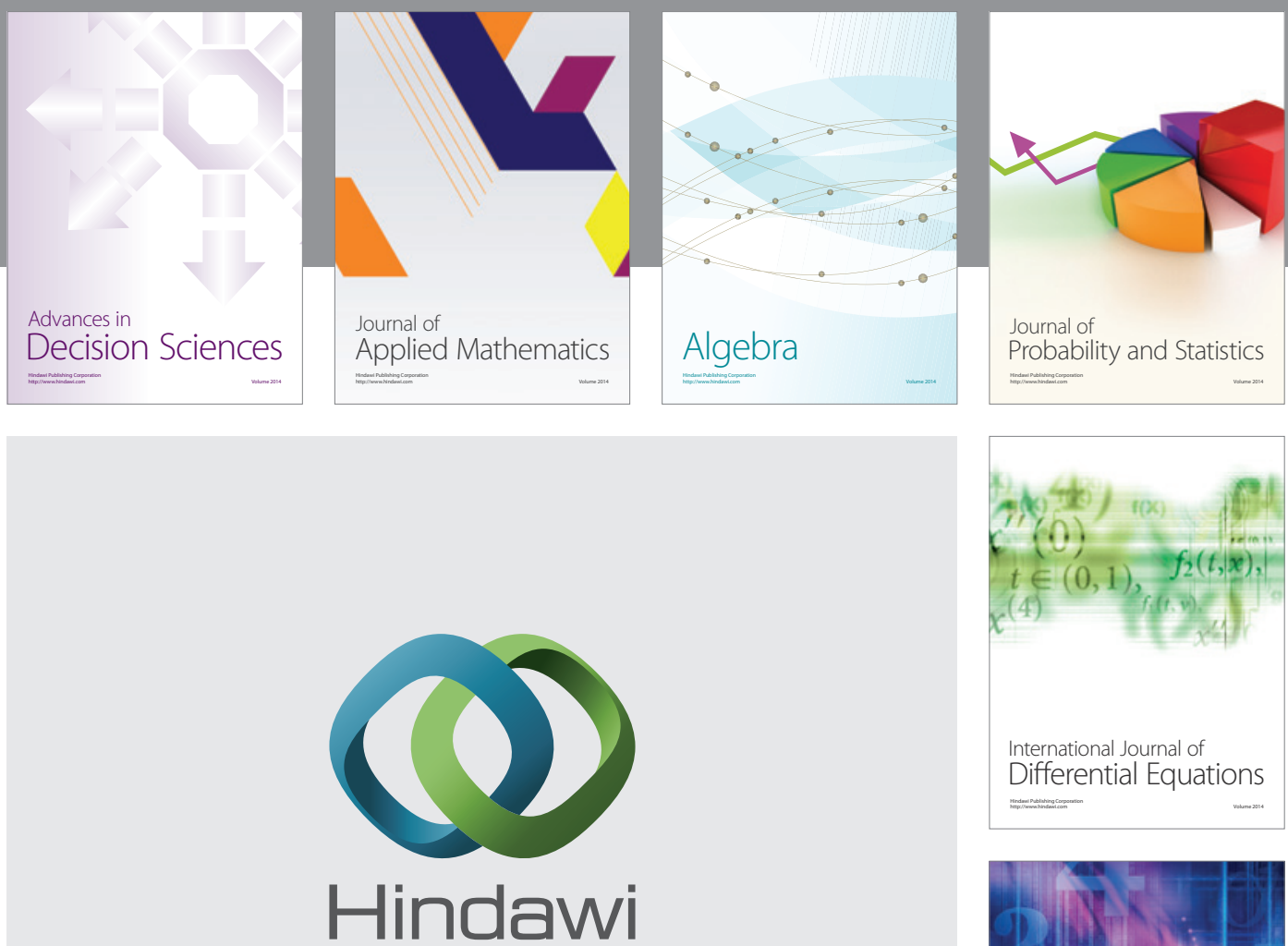

Submit your manuscripts at http://www.hindawi.com
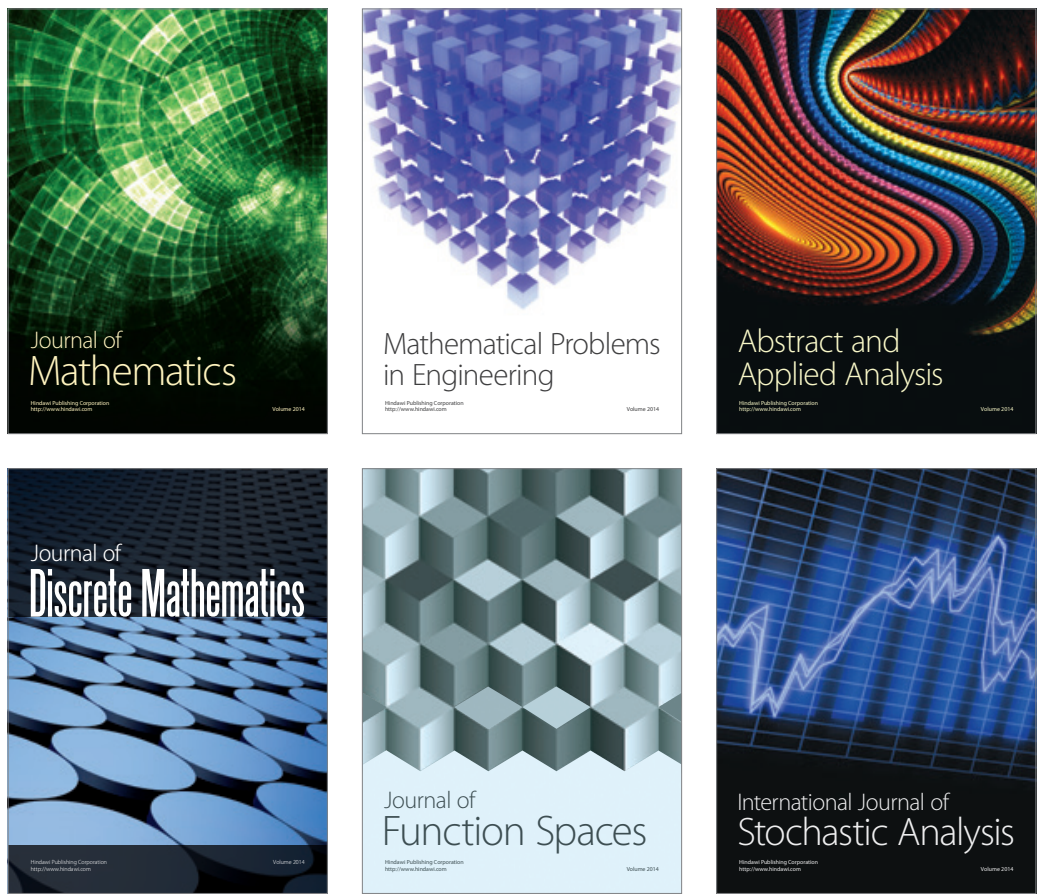

Journal of

Function Spaces

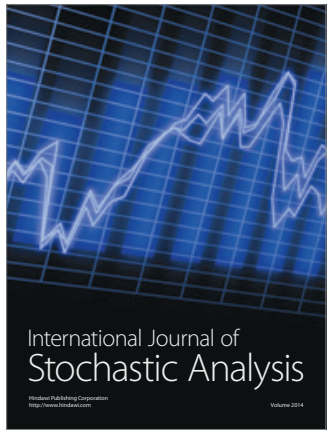

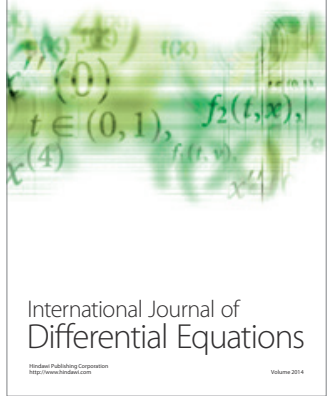
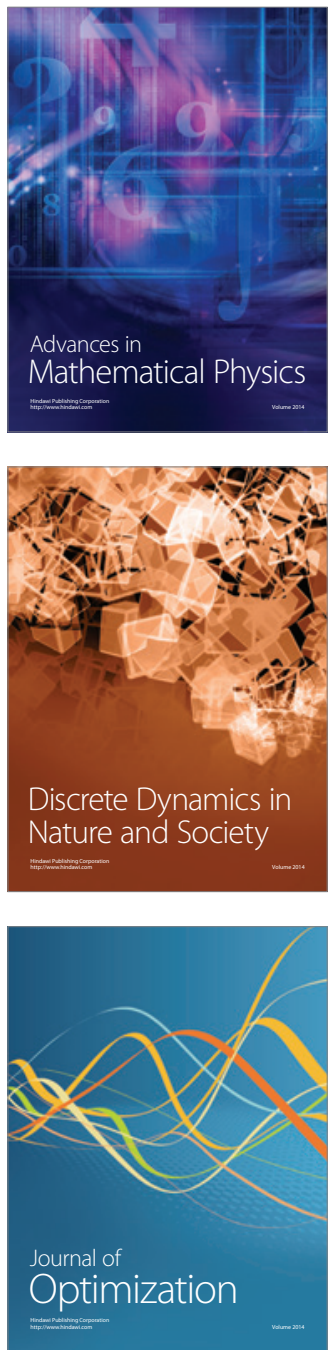